\title{
Finite element modelling and experimental characterization of an electro-thermally actuated silicon-polymer micro gripper
}

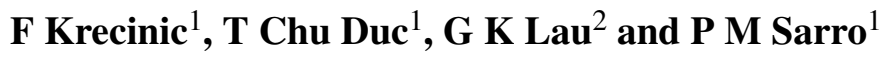 \\ ${ }^{1}$ ECTM, DIMES, Delft University of Technology, Feldmannweg 17, 2600 GB Delft, \\ The Netherlands \\ ${ }^{2}$ PME, Faculty of 3ME, Delft University of Technology, Mekelweg 2, 2628 CD Delft, \\ The Netherlands \\ E-mail: f.krecinic@tudelft.nl
}

Received 22 December 2007, in final form 24 February 2008

Published 13 May 2008

Online at stacks.iop.org/JMM/18/064007

\begin{abstract}
This paper presents simulation and experimental characterization of an electro-thermally actuated micro gripper. This micro actuator can conceptually be seen as a bi-morph structure of SU-8 and silicon, actuated by thermal expansion of the polymer. The polymer micro gripper with an embedded comb-like silicon skeleton is designed to reduce unwanted out-of-plane bending of the actuator, while offering a large gripper stroke. The temperature and displacement field of the micro gripper structure is determined using a two-dimensional finite element analysis. This analysis is compared to experimental data from steady-state and transient measurements of the integrated heater resistance, which depends on the average temperature of the actuator. The stability of the polymer actuator is evaluated by recording the transient behaviour of the actual jaw displacements. The maximum single jaw displacement of this micro gripper design is $34 \mu \mathrm{m}$ at a driving voltage of $4 \mathrm{~V}$ and an average actuator temperature of $170{ }^{\circ} \mathrm{C}$. The transient thermal response is modelled by a first-order system with a characteristic time constant of $11.1 \mathrm{~ms}$. The simulated force capability of the device is $0.57 \mathrm{mN}$ per $\mu \mathrm{m}$ jaw displacement.
\end{abstract}

(Some figures in this article are in colour only in the electronic version)

\section{Introduction}

The use of micro-electro-mechanical devices for micro object manipulation and handling has become an active research topic in the past few years. Potential application fields for these devices are, for example, micro systems assembly, micro robotics and biomedical research [1]. The micro gripper is a particular implementation of a micro manipulation scheme. Micro gripper designs based on piezo-electric [2], electrostatic [3] and electro-thermal [4] actuators have been presented in the literature. Commonly used measures of performance for these micro actuators are the force/displacement generation capability, power dissipation and the actuation speed. An advantage of the electro-thermal actuators is the relatively large strain that is generated at low voltages. Furthermore, the low response time that is characteristic of macroscopic thermal actuators is offset by scaling effects in microscopic systems. Micro scale electro-thermal actuators can therefore be driven at relatively high frequencies [5].

In this paper, a new design of an electro-thermally actuated polymer micro gripper is analysed using the finite element method (FEM) and is experimentally characterized. The model is formulated as a nonlinear, electro-thermal and thermo-elastic problem. Results of the finite element analysis are compared to experimental data in order to assess the modelling accuracy. This finite element model and the 


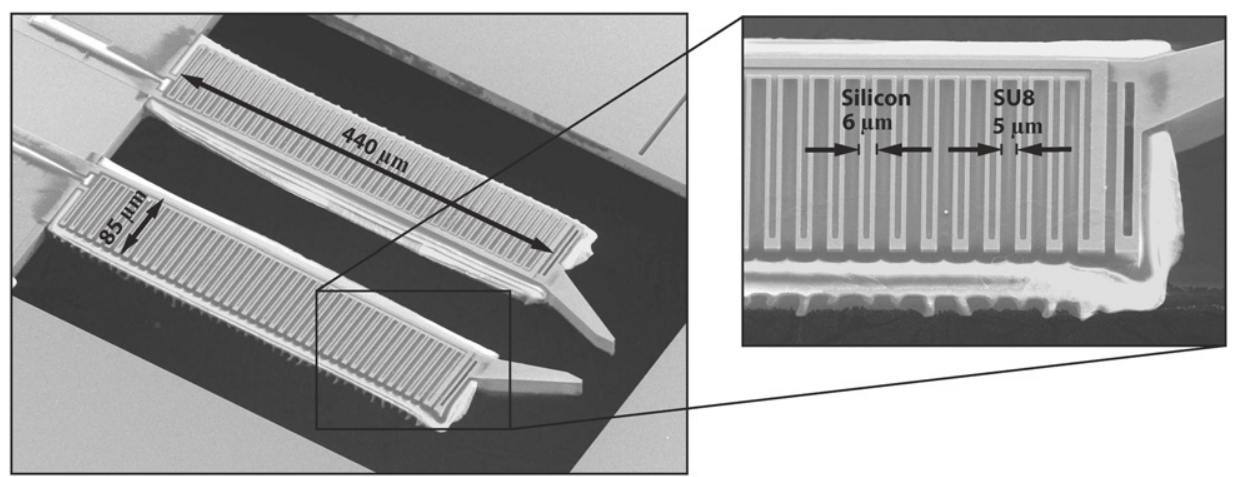

Figure 1. SEM photographs of the fabricated micro gripper. The actuator dimensions and a detail of the comb-like actuator structure are included.

experimental characterization of the device can be used as a starting point in the design of a complete micro manipulation system-on-chip, capable of more complex micro manipulation tasks and with integrated sensors for feedback control [6].

\section{Micro gripper design and fabrication}

The actuator structure of this micro gripper design consists of a comb-like silicon skeleton with a polymer material filling the space between the silicon fingers (figure 1). The polymer used is an SU-8 epoxy resin and is photo-patternable. The actuator structure bends in the in-plane direction when heated, due to the large mismatch between the coefficients of thermal expansion (CTE) of silicon and SU-8. The CTE of the SU-8 polymer is almost two orders of magnitude larger than that of the silicon, enabling the actuator to achieve large displacements at relatively low temperatures, and consequently at low power.

The comb-like structure of silicon has two main purposes in this micro gripper design. It enhances heat distribution across the thickness of the polymer layer and confines the polymer material within a high aspect-ratio structure. The enhanced heat distribution across the actuator thickness reduces temperature gradients and unwanted actuator bending in the out-of-plane direction. The confinement of the polymer material causes a concentration of the thermal strains in the in-plane direction thus enhancing the total actuator displacement [7].

The fabricated micro gripper is shown in the scanning electron microscope image in figure 1 . The silicon is etched to form a comb-like structure with $6 \mu \mathrm{m}$ wide silicon fingers and $5 \mu \mathrm{m}$ wide trenches between them. The entire actuator is $440 \mu \mathrm{m}$ long and consists of 41 silicon-polymer laterally stacked elements. The actuator is $85 \mu \mathrm{m}$ wide and $30 \mu \mathrm{m}$ thick. These dimensions have been chosen according to the analysis presented in [7] such that there is an effective confinement of the polymer material and a large transverse heat conduction coefficient, thus improving heat spreading across the insulating polymer. An integrated aluminium heater is defined on the top of the silicon structure. The heater is electrically insulated from the substrate by a layer of silicon nitride.
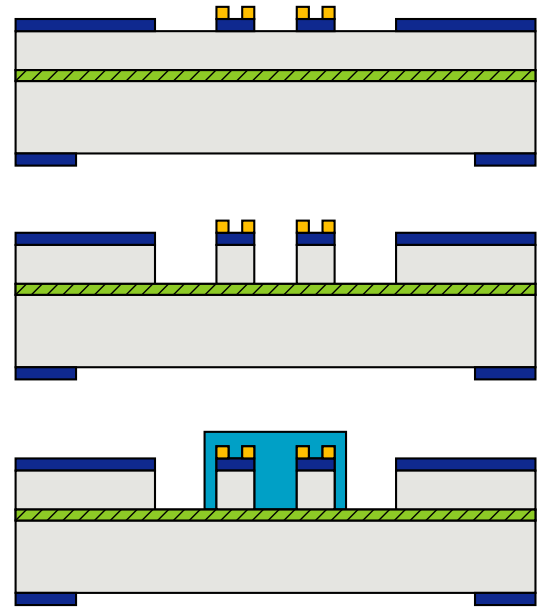

$(c)$
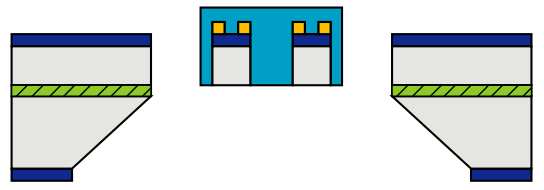

$(d)$

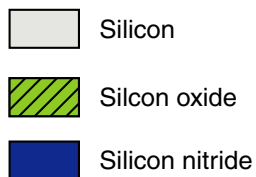

Aluminium

SU-8

Silicon nitride

Figure 2. Schematic drawing of the fabrication process: (a) silicon-nitride and aluminium patterning, (b) DRIE of silicon, (c) SU-8 deposition and patterning and (d) through-wafer release etching.

This device is fabricated using silicon on insulator (SOI) wafers and bulk micro machining techniques. The use of semiconductor processing steps and materials enables batch fabrication of the devices. The main steps of the process flow are schematically depicted in figure 2 . First, a low-stress silicon nitride layer is deposited on both sides of the wafer, followed by the sputtering of an aluminium layer on the top side only. The thin silicon nitride layer on the top side will serve as an electrical insulator in the final structure, and as a mask on the bottom side of the wafer during silicon etching in 


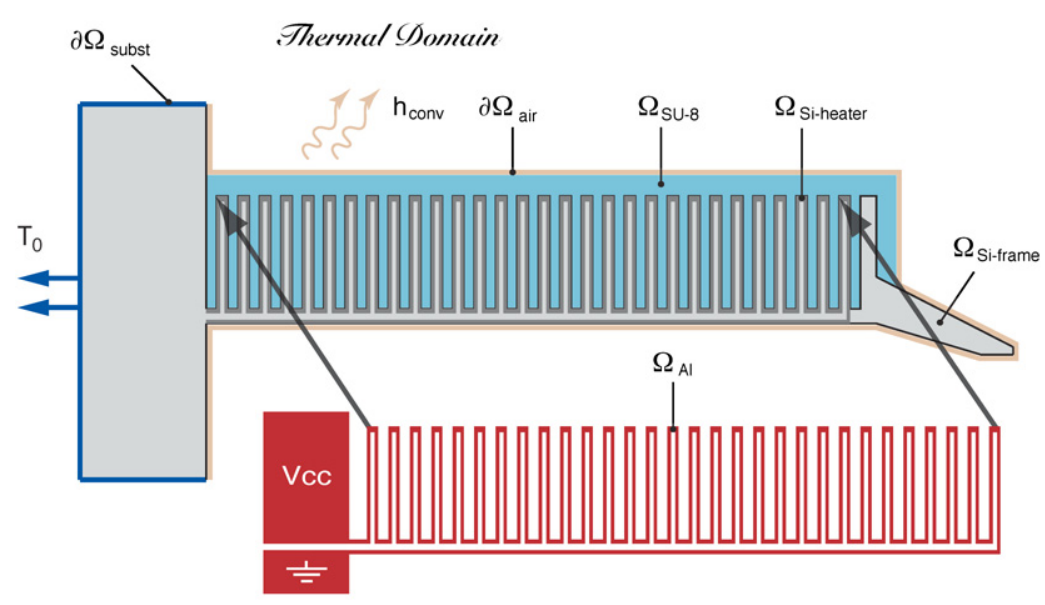

Slearical Domain

Figure 3. Sketch of the model geometry, physical domains and boundary conditions for the electro-thermal problem. The electrical potential and the temperature field in the $\Omega_{\mathrm{Al}}$ and $\Omega_{\text {Si-heater }}$ domains are mutually dependent through resistive heating effects and the temperature-dependent electrical resistivity of aluminium.

$\mathrm{KOH}$ (figure 2(a)). After the patterning of the silicon nitride and the aluminium heater layers, deep reactive ion etching (DRIE) is used to produce the high aspect-ratio, comb-like silicon structure. The buried SOI oxide is used as the stop layer to produce a uniformly etched structure (figure $2(b)$ ). The SU-8 polymer is subsequently deposited and patterned (figure 2(c)). The bulk silicon is then etched from the backside in a $\mathrm{KOH}$ solution, using the SOI oxide as stop layer. During $\mathrm{KOH}$ etching, a vacuum holder is used to protect the wafer top side from the $\mathrm{KOH}$ etchant. Finally, the structure is released by removing the buried silicon oxide layer using reactive ion etching (figure $2(d)$ ).

\section{Finite element modelling}

In order to estimate the temperature distributions, stresses and the expected strains (e.g. total jaw displacements) of the micro gripper structure, a finite element (FE) model is used. The FE analysis is also compared to experimental data, to determine the accuracy of the model. If it proves accurate enough, the model can then be used to evaluate new conceptual designs and improve the micro gripper structures.

The finite element analysis of this micro gripper structure is performed with the Comsol Multiphysics software package. Using this software, it is possible to construct a micro gripper model in which different physical domains, such as the electrical and thermal domains, are coupled. This model is formulated in two parts which are solved consecutively. First, the electro-thermal problem is formulated and solved. The thermal and electrical domains are coupled bidirectionally through resistive heating effects and the temperaturedependent change in the electrical resistivity. After obtaining the temperature field from the electro-thermal simulation, a thermo-elastic simulation is performed. The unidirectional coupling from the thermal to the elastic domain is defined by the temperature-dependent expansion of materials.

\subsection{The electro-thermal problem}

The micro gripper model geometry, boundary conditions and domains for the two-dimensional electro-thermal problem are shown in figure 3. Thermal conductivity of the silicon plates is almost two orders of magnitude larger than that of the surrounding SU-8 or air. The temperature gradient throughout the actuator thickness is therefore very small, and a two-dimensional simplification of the full three-dimensional actuator model of [8] is used in this analysis. However, the out-of-plane heat transfer to the surrounding air by conduction and convection is significant and must be modelled by temperature-dependent out-of-plane heat flux factors in the two-dimensional heat equation. The electrical domain analysis can be simplified to two dimensions as well, because electrical current flow is confined to the thin aluminium heater.

The mathematical formulation of the electro-thermal problem for this structure consists of a combination of the general heat equation (equations $(1 a)$ and $(1 b)$ ) and the electrostatic equation (equation $(1 c)$ ):

$$
\begin{gathered}
\nabla \cdot\left(-d_{\mathrm{Si}} k_{\mathrm{Si}} \nabla T\right) \\
= \begin{cases}d_{\mathrm{Si}} Q_{\text {electric }}+h_{\mathrm{vert}}\left(T_{0}-T\right) & \text { on } \Omega_{\mathrm{Si} \text {-heater }} \\
h_{\mathrm{vert}}\left(T_{0}-T\right) & \text { on } \Omega_{\mathrm{Si}-\text { frame }}\end{cases} \\
\nabla \cdot\left(-d_{\mathrm{Si}} k_{\mathrm{SU}-8} \nabla T\right)=h_{\mathrm{vert}}\left(T_{0}-T\right) \\
-\nabla \cdot d_{\mathrm{Al}}\left(\sigma_{\mathrm{Al}} \nabla V\right)=0 \quad \text { on } \Omega_{\mathrm{SU}-8}
\end{gathered}
$$

The geometric domains $\Omega_{\text {Si-frame }}, \Omega_{\text {Si-heater }}, \Omega_{\mathrm{SU}-8}$ and $\Omega_{\mathrm{Al}}$ on which these equations are defined and the boundary conditions, $\partial \Omega_{\text {subst }}$ and $\partial \Omega_{\text {air }}$, for the problem are indicated in figure 3 . Solving this set of equations yields the temperature and potential fields, $T$ and $V$, respectively. The terms $k_{\mathrm{Si}}$ and $k_{\mathrm{SU}-8}$ in $(1 a)$ and $(1 b)$ are the thermal conductivities of silicon and SU-8, respectively (table 1 ). The temperature dependence of the thermal conductivity of silicon is taken into account and a linear interpolation function is used to obtain intermediate 


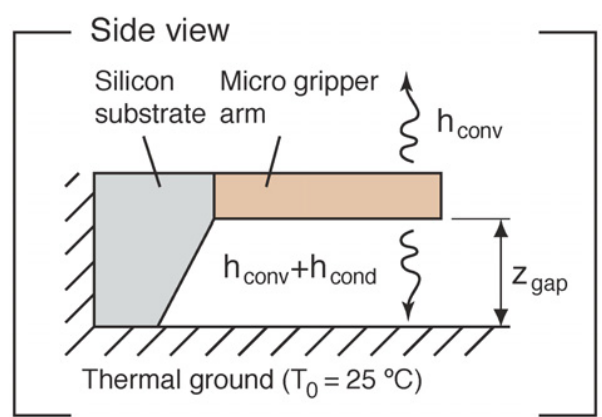

Figure 4. Sketch of the out-of-plane heat transfer modelling, illustrating the heat transfers through the air by convection and conduction from the top and bottom sides of the gripper.

Table 1. Thermal conductivity of silicon [9], air [10] and SU-8 [11].

\begin{tabular}{lllll}
\hline $\begin{array}{l}\text { Thermal conductivity } \\
\left(\mathrm{W} \mathrm{mK}^{-1}\right)\end{array}$ & $300 \mathrm{~K}$ & $400 \mathrm{~K}$ & $500 \mathrm{~K}$ & $600 \mathrm{~K}$ \\
\hline$k_{\mathrm{Si}}$ & 148 & 119 & 98.9 & 76.2 \\
$k_{\text {Air }}$ & 0.0263 & 0.0338 & 0.0407 & 0.0469 \\
$k_{\mathrm{SU}-8}$ & 0.2 & & & \\
\hline
\end{tabular}

Table 2. Electrical model properties.

\begin{tabular}{ll}
\hline Parameter & Value \\
\hline $\begin{array}{c}\text { Aluminium electrical resistivity at } \\
\text { room temperature }\left(\rho_{0}\right)[9]\end{array}$ & $3.1 \times 10^{-8} \Omega \mathrm{m}$ \\
$\begin{array}{c}\text { Aluminium temperature coefficient } \\
\text { of resistance }(\alpha)[9]\end{array}$ & $4.13 \times 10^{-3} \mathrm{~K}^{-1}$ \\
Aluminium thickness $\left(d_{\mathrm{Al}}\right)$ & $600 \mathrm{~nm}$ \\
Silicon thickness $\left(d_{\mathrm{Si}}\right)$ & $30 \mu \mathrm{m}$ \\
\hline
\end{tabular}

values during the simulation. The thermal conductivity of SU8 is assumed to be constant for this temperature range. The heat source factor $Q_{\text {electric }}$ in $(1 a)$ represents the heat source power density injected into the $\Omega_{\text {Si-heater }}$ silicon structure by the aluminium heater, while $d_{\mathrm{Al}}$ and $\sigma_{\mathrm{Al}}$ are the aluminium layer thickness and its electrical resistivity, respectively (table 2).

The out-of-plane heat flux coefficient $h_{\text {vert }}$ in (1a) models the temperature-dependent heat transfer from the actuator top and bottom sides (figure 4). The reference temperature $T_{0}$ is the room temperature, i.e. $25{ }^{\circ} \mathrm{C}$. The coefficient consists of convective and conductive heat transfers through the surrounding air and can be written as

$$
h_{\mathrm{vert}}=2 h_{\mathrm{conv}}+h_{\mathrm{cond}} \text {. }
$$

The $h_{\text {cond }}$ coefficient models the heat conduction through a thin air layer between the gripper bottom side and the heat sink (i.e. wafer test support). It can be approximated by

$$
h_{\text {cond }}=\frac{k_{\text {Air }}}{z_{\text {gap }}},
$$

where $k_{\text {Air }}$ is the temperature-dependent thermal conductivity of air (table 1) and $z_{\mathrm{gap}}$ is the air layer thickness of $470 \mu \mathrm{m}$.

The convection coefficient $h_{\text {conv }}$ is obtained from correlating equations for free convection from a horizontal cylinder by Churchill and Chu [12]. When evaluated on this

\begin{tabular}{|c|c|c|}
\hline Parameter & Silicon [14] & SU-8 [11] \\
\hline Young's modulus (GPa) & 130 & 3.2 \\
\hline Poisson's ratio & 0.26 & 0.33 \\
\hline $\begin{array}{l}\text { Coefficient of thermal } \\
\text { expansion }\left(\mathrm{ppm}^{\circ} \mathrm{C}^{-1}\right)\end{array}$ & 2.6 & 150.7 \\
\hline
\end{tabular}

Table 3. Thermo-elastic material properties.

structure, following the procedures of [8], these equations result in the expression

$$
h_{\mathrm{conv}}=\frac{0.36 k_{\mathrm{Air}}}{D}
$$

where $D$ is the effective diameter of the gripper structure. The effective diameter is defined as $4 A / P$, in which $A$ is the cross-sectional area and $P$ is the perimeter over the section. The value of $D$ for our structure is $44.3 \mu \mathrm{m}$. The convective heat transfer takes place at the top and bottom sides of the structure, leading to the factor of 2 in (2).

The factors $Q_{\text {electric }}$ and $\sigma_{\mathrm{Al}}$ define the coupling between thermal and electrical mode equations. The heating power density $Q_{\text {electric }}$ is derived from the power that is dissipated in the conductor. It is a function of the heater layer conductivity $\sigma_{\mathrm{Al}}$, the electric field $\mathbf{E}$ and the ratio of the silicon and aluminium layer thicknesses (equation $(5 a)$ ). The electrical conductivity of the heater is dependent on the temperature of the silicon structure and the temperature coefficient of resistance of aluminium, $\alpha_{\mathrm{Al}}$ (equation $(5 b)$ ). The values of the parameters $\alpha_{\mathrm{Al}}, d_{\mathrm{Si}}, d_{\mathrm{Al}}$ and $\rho_{0}$, used in the electrical domain model, are listed in table 2:

$$
\begin{gathered}
Q_{\text {electric }}=\frac{d_{\mathrm{Al}}}{d_{\mathrm{Si}}} \sigma_{\mathrm{Al}}|\mathbf{E}|^{2}, \\
\sigma_{\mathrm{Al}}=\frac{1}{\rho_{0}\left[1+\alpha_{\mathrm{Al}}\left(T-T_{0}\right)\right]} .
\end{gathered}
$$

The thermal boundary conditions are formulated as a temperature-dependent heat flux condition on the gripper perimeter and a fixed temperature condition at the substrate anchor [13]. The temperature-dependent heat flux condition at the $\partial \Omega_{\text {air }}$ boundary represents convective heat transfer from the actuator sides to the surrounding air and is modelled by the $h_{\text {conv }}$ coefficient from (4). The anchor boundary $\partial \Omega_{\text {subst }}$ is fixed at the room temperature $T_{0}$ :

$$
\begin{aligned}
-\mathbf{n} \cdot(k \nabla T) & =h_{\text {conv }}\left(T-T_{0}\right) \quad \text { at } \quad \partial \Omega_{\text {air }} \\
T & =T_{0} \quad \text { at } \partial \Omega_{\text {subst }} .
\end{aligned}
$$

Electrical boundary conditions are shown at the bottom side of figure 3. A fixed potential is applied at the contact pads, while applying an electrical insulation condition on the perimeter of the domain.

\subsection{The thermo-elastic problem}

The temperature field that results from the electro-thermal simulation is used in a two-dimensional plane strain simulation, to obtain the micro gripper jaw displacement resulting from the thermal expansion of the polymer. Thermo-elastic material properties used in this simulation are listed in table 3 . The simulated force capability of the device is $0.57 \mathrm{mN}$ per $\mu \mathrm{m}$ jaw displacement. 


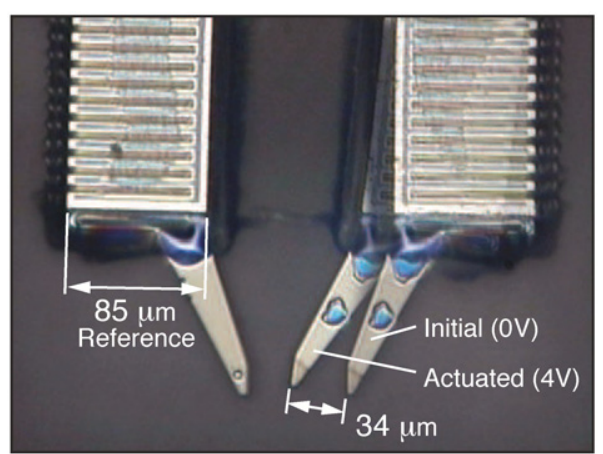

(a)

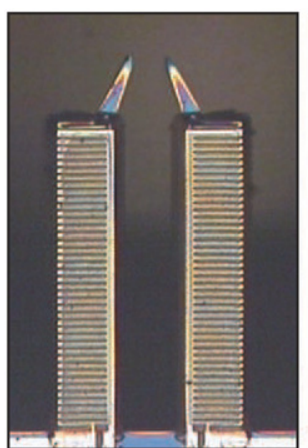

(b)

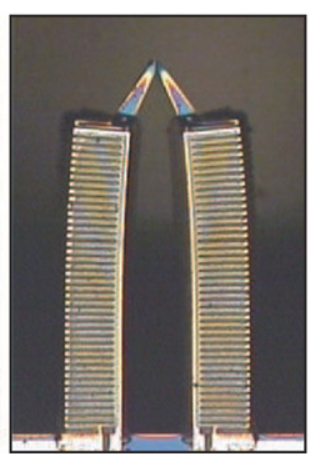

Figure 5. Microscope images of the micro gripper: $(a)$ actual jaw displacements are extracted from images before and after actuation; $(b)$ an image sequence showing the closing of micro gripper jaws on actuation of both arms.
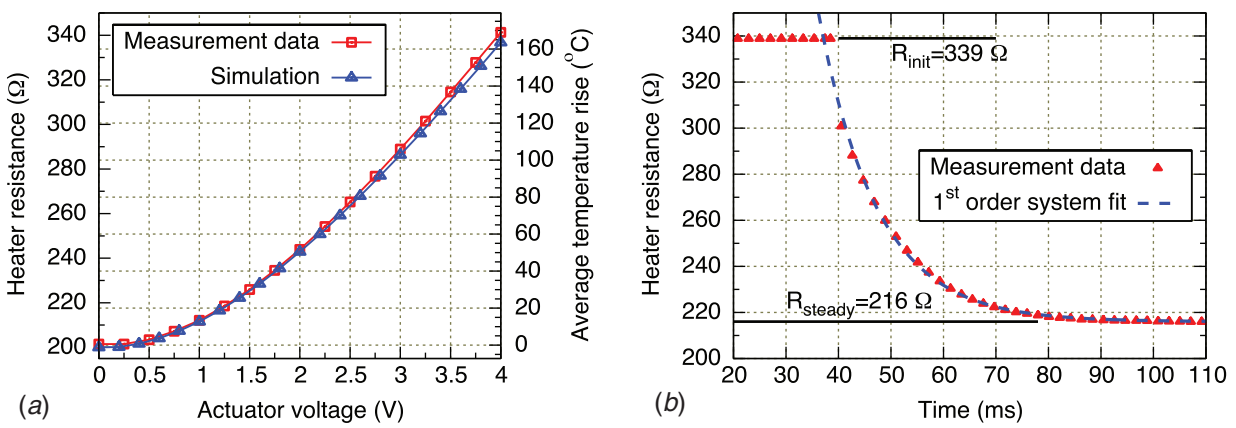

Figure 6. Heater resistance measurements and simulation results: (a) steady-state and $(b)$ transient response.

\section{Experimental results}

In this section, the experimental methods and setups that are used to characterize the micro gripper are discussed first, followed by the experimental results. The purpose of these experiments is to obtain information on the average temperature rise in the actuator structure and the micro gripper jaw displacements.

\subsection{Experimental setup}

The jaw displacement measurements are performed with a probe station equipped with a microscope and a digital camera. Images of the micro scale structure that are taken with the mounted camera are processed with a software package in order to extract actual jaw displacements. This process is shown in figure 5(a). The reference image of the micro gripper and the image of the structure after actuation are compared to each other and a relative displacement of the gripper jaws is obtained. The relative displacement is converted to the actual displacement by using a known structural dimension, such as the actuator width, as a reference. This measurement method has a displacement resolution of $1.5 \mu \mathrm{m}$. As is shown in the image sequence in figure $5(b)$, the micro gripper is capable of fully closing the $45 \mu \mathrm{m}$ gap between the jaws and can be used to grab micro scale objects [6].

The jaw displacement measurement technique is used in two experiments. In the first experiment, a photo is taken just before and just after the application of an actuation voltage. The initial thermal expansion of the polymer material is the cause of the micro gripper jaw displacement and takes place as rapidly as the change in the temperature of the structure. However, heating of the polymer material can also have an effect on its mechanical and chemical properties. To evaluate the stability of the polymer actuator, a second experiment is performed in which the gripper is continuously actuated at the maximum voltage for a period of $5 \mathrm{~min}$, while recording the jaw displacements on video. Images that are extracted from this video recording are processed to extract the jaw displacements and monitor their stability.

The average temperature of the actuator can be monitored using the temperature-dependent change in the electrical resistance of the aluminium heater. The heater resistance measurement is performed with the HP4156A semiconductor parameter analyser. Two types of measurements are performed: sweep measurements, indicating steady-state temperature changes as a function of the actuator voltage, and sampling measurements, for the characterization of the heating and cooling dynamics. The sweep measurement time frame is chosen long enough such that the actuator has reached a steady-state temperature. For the sampling measurement, the emphasis is on measuring the speed with which the structure reaches the steady-state temperature and the dynamics of the thermal process. 

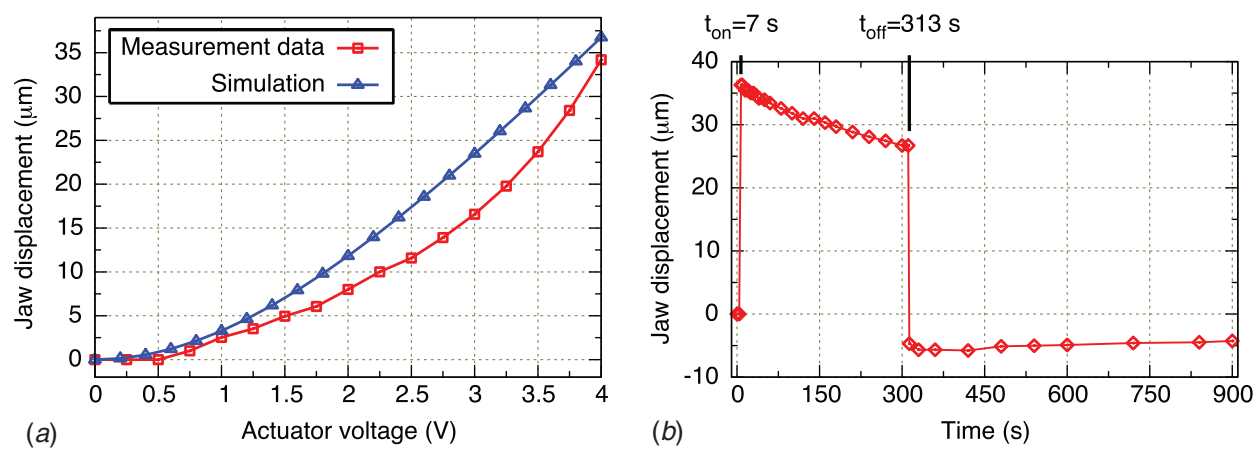

Figure 7. Jaw displacement measurements and simulations: $(a)$ initial displacements versus voltage and $(b)$ displacement versus time.

\subsection{Results}

The actuator voltage sweep measurement and steady-state electro-thermal simulation results are plotted in figure $6(a)$. The maximum average temperature rise on the structure is $170^{\circ} \mathrm{C}$ at an actuation voltage of $4 \mathrm{~V}$. There is a good agreement between the simulation and the measurement data, with a deviation of only $1 \%$ at the highest actuation voltages.

The results of sampling measurements are shown in figure $6(b)$. The graph shows the transient change in resistance on deactivation of the actuator by switching of the input voltage from $4 \mathrm{~V}$ to $1 \mathrm{~V}$, since the dominant time constant of this system is actually the cooling time. The thermal actuator transient response can be modelled accurately by a first-order system. Fitting of the first-order system response to measurement data results in a first-order system with a characteristic time constant of $11.1 \mathrm{~ms}$.

Figure 7(a) shows the initial jaw displacements versus the actuation voltage. The maximum single jaw displacement of $34 \mu \mathrm{m}$ is reached at a total power consumption of $46.9 \mathrm{~mW}$ (figure 7(a)). The micro gripper jaws remained in focus after actuation. Considering the vertical displacement resolution obtained from the depth of focus of the used microscope objective, it is concluded that any out-of-plane bending is less than $1 \mu \mathrm{m}$.

The simulated jaw displacement overestimates the actual displacement by up to $6.5 \mu \mathrm{m}$. It is possible that the simulated temperature profile does not match the real temperature, causing a deviation between the simulated and measured jaw displacements. However, assuming a linear relation between the temperature and jaw displacement, this would mean that the measured and simulated average temperatures of the actuator are off by some $20 \%$ with respect to the actual temperature, which is rather large compared to the accuracy of temperature measurements and simulations. This deviation is therefore thought to be caused by the uncertainty in thermo-elastic material properties of the SU-8, which tend to be sensitive to the processing conditions during fabrication, and the polymer temperature during actuator operation [15].

The measured jaw displacement versus time can be seen in figure 7(b). The actuator voltage is switched from 0 to $4 \mathrm{~V}$ at $t_{\text {on }}$ and held constant for a period of $5 \mathrm{~min}$, until it is switched back to $0 \mathrm{~V}$ at $t_{\text {off }}$. The jaw displacement does not remain stable during actuation and is seen to reduce gradually by some $10 \mu \mathrm{m}$ during the $5 \mathrm{~min}$ actuation period. The gripper also does not return entirely to its starting position after the actuation, although there is a small, but slow, recovery towards the initial jaw position.

These phenomena are believed to be due to the effects of elevated temperature on the polymer material. The irreversible backward bending after actuation may be the result of induced cure shrinkage and pre-stress formation caused by actuator temperatures exceeding the glass transition temperature of the polymer [15]. The visco-elastic behaviour of the polymer material, which becomes more pronounced at high temperatures, is also a possible factor contributing to the gradual relaxation of the jaw displacement during actuation [16].

\section{Conclusions}

The micro gripper presented in this paper features an electro-thermal actuator design achieving large displacements at low driving voltages. The relatively low operating temperature required also means a lower power consumption. The comb-like silicon structure of the gripper serves to improve heat distribution across the polymer layer and to confine the polymer material, enhancing the in-plane actuator displacements. Standard semiconductor processing and materials are used in the micro gripper fabrication process, making batch fabrication of the devices possible.

A two-dimensional finite element model is used to analyse the thermal, electrical and mechanical behaviour of the micro gripper. The model is formulated as an electro-thermal problem and a thermo-elastic problem that are solved consecutively. Solving the electro-thermal problem results in a temperature field, which is then used to determine the jaw displacements resulting from the thermal expansion of the polymer material.

Experiments have been performed to characterize the micro gripper and verify the finite element modelling. Steady-state electro-thermal measurements involving the heater resistance are in good agreement with the simulations, showing a deviation of only $1 \%$ at the highest actuation voltages. The transient electro-thermal measurement data 
show that the thermal process can be modelled as a first-order system with a characteristic time constant of $11.1 \mathrm{~ms}$.

Jaw displacement measurements have also been performed. A reduction of the gripper displacement of up to $10 \mu \mathrm{m}$ during an actuation period of $5 \mathrm{~min}$ has been observed. It is believed that the polymer is affected by the elevated temperatures, which can cause chemical, visco-elastic and mechanical changes in the material.

\section{Acknowledgments}

The exact design of the comb-finger was claimed in a Dutch patent (G K Lau et al, application no 2000209 filed on 13 September 2006). The authors would like to acknowledge the whole DIMES IC Process group for technical support, Mr P J F Swart and Mr J Wei for their help on the fabrication of devices and the experimental setup.

\section{References}

[1] Cecil J, Vasquez D and Powell D 2005 A review of gripping and manipulation techniques for micro-assembly applications Int. J. Prod. Res. 43 819-28

[2] Haddab Y, Chaillet N and Bourjault A 2000 A microgripper using smart piezoelectric actuators Proc. IEEE/RSJ Int. Conf. on Intelligent Robots and Systems vol 1 pp 659-64

[3] Beyeler F, Neild A, Oberti S, Bell D J, Sun Y, Dual J and Nelson B J 2007 Monolithically fabricated microgripper with integrated force sensor for manipulating microobjects and biological cells aligned in an ultrasonic field J. Microelectromech. Sys. 16 7-15

[4] Chronis N and Lee L P 2005 Electrothermally activated SU-8 microgripper for single cell manipulation in solution J. Microelectromech. Sys. $14857-63$

[5] Wood D, Burdess J S and Harris A J 1998 Actuators and their mechanisms in microengineering Eng. Sci. Educ. J. 7 19-27
[6] Chu Duc T, Lau G K, Creemer J F and Sarro P M 2008 Electrothermal microgripper with large jaw displacement and integrated force sensors Proc. 21st IEEE Int. Conf. on MEMS (Tucson, AZ, USA, January 13-17, 2008) pp 519-22

[7] Lau G K, Goosen J F L, van Keulen F, Chu Duc T and Sarro P M 2007 A powerful polymeric thermal microactuator with embedded silicon microstructure Appl. Phys. Lett. 90214103

[8] Lau G K, Chu Duc T, Goosen J F L, Sarro P M and van Keulen F 2007 In-plane thermal unimorph using confined polymers J. Micromech. Microeng. 17 1-10

[9] Lide D R (ed) 2006 Thermal conductivity of metals and semiconductors as a function of temperature $C R C$ Handbook of Chemistry and Physics 87th edn (Boca Raton, FL: Taylor and Francis) internet version p 12.219

[10] Incropera F P and DeWitt D P 1996 Thermophysical properties of gases at atmospheric pressure, Appendix A Table A.4 Fundamentals of Heat and Mass Transfer 4th edn (New York: Wiley) p 839

[11] Feng R and Farris R J 2002 The characterization of thermal and elastic constants for an epoxy photoresist su8 coating J. Mater. Sci. 37 4793-9

[12] Churchill S W and Chu H 1975 Correlating equations for laminar and turbulent free convection from a horizontal cylinder Int. J. Heat Mass Transfer 18 1049-53

[13] Mankame N D and Ananthasuresh G K 2001 Comprehensive thermal modelling and characterization of an electro-thermal-compliant microactuator J. Micromech. Microeng. 11 452-62

[14] Frederikse P R 2006 Elastic constants of single crystals CRC Handbook of Chemistry and Physics 87th edn (Boca Raton, FL: Taylor and Francis) internet version p 12.77

[15] Feng R and Farris R J 2003 Influence of processing conditions on the thermal and mechanical properties of SU8 negative photoresist coatings J. Micromech. Microeng. 13 80-8

[16] Namazu T, Inoue S, Takio K, Fujita T, Maenaka K and Koterazawa K 2005 Visco-elastic properties of micron-thick SU-8 polymers measured by two different types of uniaxial tensile tests MEMS 2005, 18th IEEE Int. Conf. pp 447-50 